\title{
Resultados del Convenio de Cooperación No 17 -16- 062- 022CE entre el Instituto de Investigación de Recursos Biológicos Alexander von Humboldt (IAvH) y la Sociedad Colombiana de Mastozoología (SCMas)
}

\section{Sociedad Colombiana de Mastozoología ${ }^{1}$}

mastozoologiacolombia@gmail.com

Consecuentes con el carácter misional de la Estrategia del Inventario Nacional de la Biodiversidad (ENIBIO), la cual pretende el intercambio de información y disponibilidad de datos que apuntan a fortalecer el flujo permanente de la información sobre la biodiversidad entre todas las instituciones involucradas en su estudio, de acuerdo con los parámetros, estándares y liderazgo del Sistema de Información sobre Biodiversidad de Colombia - SiB, el Instituto Alexander von Humboldt (IAvH) y la Sociedad Colombiana de Mastozoología (SCMas) unieron esfuerzos técnicos, administrativos y financieros, para sumar al inventario de la biodiversidad nacional información referente a los mamíferos que en el país ocurren, a través del convenio de cooperación No. 17 -16- 062- 022CE.

Los productos de dicho convenio serán descritos en la presente correspondencia y abarcan la actualización de la última lista de referencia de especies (Ramírez-Chaves et al. 2016), la realización de 50 fichas de especies y la publicación de más de 3500 registros biológicos de mamíferos en la plataforma SiB bajo el estándar Darwin Core. Empleamos como herramienta de trabajo un proceso concertado, colaborativo y dinámico entre Universidades públicas y privadas, Colecciones Biológicas en cabeza de sus Curadores, Organizaciones No Gubernamentales, becarios y socios de la SCMas.

Lista de referencia de especies de mamíferos de Colombia

A partir de los listados publicados por Solari et al. (2013), Suárez-Castro y Ramírez-Chaves (2014), y Ramírez-Chaves et. al. (2016), se actualizó el listado de especies de Colombia. Los datos de la nueva lista fueron consignados en la plantilla v.3.1 (DwC) del SiB y validados por la autoridad taxonómica Héctor Ramírez Chaves. El área geográfica incluida fue todo el territorio colombiano, incluyendo sus territorios insulares, no tuvo escala temporal y la escala filogenética fue a nivel de "especie". Ademas, se complementó la información con los metadatos siguiendo los lineamientos de GBIF y se realizó la validación de los nombres científicos a partir del Catalogue of Life.

La actualización de la lista de especies de Ramírez-Chaves et al. (2016), adicionó 10 nuevas especies, sumando un total de 528. El estado taxonómico de las especies incluyó un 84,6\% compuesto por especies validadas por las listas internacionales. Tres especies de los géneros Gracilinanus, Monodelphis y Carollia, están en proceso de descripción, por lo que éstas se reportaron a nivel de "genero". La lista actual izada de mamíferos para Col ombia cuenta con representantes de 14 órdenes, 44 familias y 214 géneros. Los órdenes Chiroptera y Rodentia comprenden más del 50\% de las especies de país. De éstas, un total de 58 especies se reportan como endémicas, esto es, que habitan exclusivamente el territorio colombiano (Tabla 1).

De otro lado, considerando los criterios de amenaza de la Unión Internacional para la Conservación de la Naturaleza (UICN), encontramos que en el país el 86\% de las especies no han sido evaluadas, están categorizadas como Deficientes de Datos (DD) o como Preocupación Menor (LC); el 14\% se encuentra bajo una categoría de riesgo (Vulnerable, Amenazada o Críticamente Amenazada; Figura 1). De acuerdo con la Convención sobre el Comercio Internacional de Especies Amenazadas de Fauna y Flora Silvestres (CITES), el 16,8\% de las especies son objeto de comercialización en el país; esto equivale a 89 especies, estando algunas de las mismas incluidas en más de un apéndice a nivel mundial (Tabla 2).

Por último, la validación de los nombres científicos a través del Catalogue of Life (http://www.catalogueoflife.org), reportó 457 nombres de especies de mamíferos como válidos y 71 nombres no están incluidos en este catálogo. La lista se encuentra publicada en el enlace http://www.gbif.org/dataset/e8b9ed9b-f715-4eac-ae24-772fbf40d7ae (doi: 10.15472/k11whs).

\footnotetext{
Mammalogy Notes | Notas Mastozoológicas

Sociedad Colombiana de Mastozoología

Vol. 4 Num. 2| 2018
} 
Tabla 1. Riqueza y endemismo de los órdenes de mamíferos presentes en Colombia

\begin{tabular}{lcccccc}
\hline \hline \multicolumn{1}{c}{ Orden } & Familia & Género & Especies & $\begin{array}{c}\text { Porcentaje } \\
\text { especies }\end{array}$ & $\begin{array}{c}\text { Especies } \\
\text { endémicas }\end{array}$ & $\begin{array}{c}\% \\
\text { endémica }\end{array}$ \\
\hline Artiodactyla & 1 & 3 & 10 & 1,89 & & \\
Carnivora & 6 & 24 & 34 & 6,44 & & 13,79 \\
Cetacea & 5 & 19 & 30 & 5,68 & & \\
Chiroptera & 6 & 70 & 208 & 39,58 & 8 & \\
Cingulata & 1 & 3 & 6 & 1,14 & & \\
Didelphimorphia & 1 & 13 & 40 & 7,58 & 5 & \\
Lagomorpha & 1 & 1 & 4 & 0,76 & & \\
Paucituberculata & 1 & 1 & 2 & 0,38 & & \\
Perissodactyla & 1 & 3 & 5 & 0,94 & & \\
Pilosa & 4 & 5 & 7 & 1,33 & & \\
Primates & 5 & 15 & 40 & $7,58,45$ & 8,62 \\
Rodentia & 10 & 55 & 132 & 25,00 & 31 & 5 \\
Soricomorpha & 1 & 1 & 7 & 1,33 & & \\
Sirenia & 1 & 1 & 2 & 0,38 & & \\
\hline
\end{tabular}

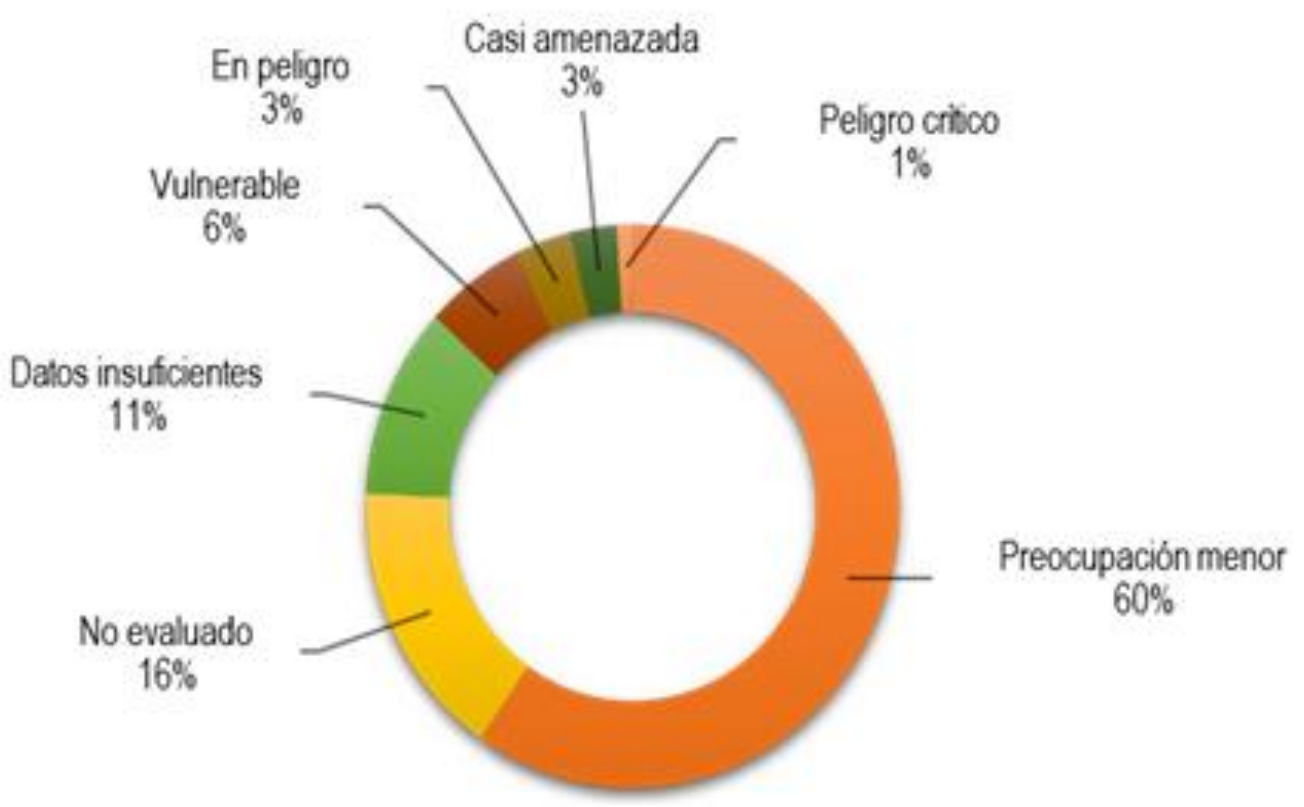

Figura 1. Porcentaje de las categorías de amenaza según IUCN para los mamíferos de Colombia

Tabla 2. Número de especies mamíferos de Colombia en los apéndices CITES.

\begin{tabular}{cc}
\hline \hline Apéndice CITES & Número de especies \\
\hline I & 29 \\
II & 53 \\
III & 2 \\
NC & 2 \\
\hline \hline
\end{tabular}

Fichas de Especies de Mamíferos

En palabras de Dairo Escobar, Coordinador de SiB Colombia, “El Catalogo del Sistema de Información en Biodiversidad tiene un modelo conceptual definido frente a cómo se publica, qué se publica y cómo se hace la atribución, entre otros. Este modelo aún es un documento de trabajo que va evolucionando en la medida que se avanza con el desarrollo del mismo. En éste, los contenidos de las fichas de especies son estructurados bajo el estándar Plinian Core (https://github.com/tdwg/PlinianCore/wiki), estructura estándar mundial para representar completamente la información de una especie en una variedad de escenarios de un taxón". Siguiendo los elementos definidos en la plantilla "tcd-c-sib-20" del sistema SiB, el cual contiene la jerarquía anidada y las definiciones para cada elemento, reunimos 65 fichas de especies (Tabla

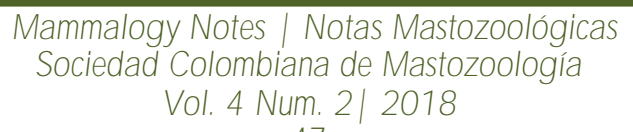




\section{Correspondencia | Correspondencia}

3), en las que intervinieron 66 autores, pertenecientes a 29 instituciones. Las fichas fueron elaboradas de manera voluntaria por socios activos y las instituciones que participaron en el desarrollo del convenio. La revisión científica de las fichas estuvo a cargo por una comisión de arbitraje del Comité Editorial de la SCMas, conformado por 16 socios especialistas. Para la vinculación de las imágenes que acompañaron las fichas de especies (mapeo), se creó un sitio web en Flickr nombrado “Mamíferos de Colombia” (https://www.flickr.com/photos/154330806@N06/), en el cual se subieron 167 fotografías aportadas por los socios.

Tabla 3. Fichas construidas de las especies de mamíferos de Colombia.

\begin{tabular}{|c|c|c|c|c|c|}
\hline Orden & Familia & Especie & Orden & Familia & Especie \\
\hline Didelphimorphia & Didelphidae & Marmosa isthmica & \multirow{12}{*}{ Carnivora } & \multirow{6}{*}{ Felidae } & Leopardus pardalis \\
\hline \multirow{6}{*}{ Cingulata } & \multirow{6}{*}{ Dasypodidae } & Cabassous centralis & & & Leopardus tigrinus \\
\hline & & Cabassous unicinctus & & & Leopardus wiedii \\
\hline & & Caenolestes fuliginossus & & & Puma concolor \\
\hline & & Dasypus kappleri & & & Puma yagouaroundi \\
\hline & & Dasypus novemcinctus & & & Panthera onca \\
\hline & & $\begin{array}{l}\text { Dasypus sabanicola } \\
\text { Priodontes maximus }\end{array}$ & & Canidae & $\begin{array}{l}\text { Cerdocyon thous } \\
\text { Speothos venaticus }\end{array}$ \\
\hline \multirow{4}{*}{ Pilosa } & Bradypodidae & Bradypus variegatus & & \multirow{3}{*}{ Mustelidae } & Eira barbara \\
\hline & Cyclopedidae & Cyclopes didactylus & & & Galictis vittata \\
\hline & Myrmecophagidae & $\begin{array}{l}\text { Myrmecophaga tridactyla } \\
\text { Tamandua mexicana }\end{array}$ & & & $\begin{array}{l}\text { Lontra longicaudis } \\
\text { Pteronura brasiliensis }\end{array}$ \\
\hline & Megalonychidae & Choloepus hoffmanni & & \multirow{2}{*}{ Procyonidae } & Bassaricyon neblina \\
\hline Sirenia & Trichechidae & Trichechus inunguis & & & Procyon lotor \\
\hline \multirow{18}{*}{ Chiroptera } & Emballonuridae & Rhynchonycteris naso & \multirow{2}{*}{ Perissodactyla } & \multirow{2}{*}{ Tapiridae } & Tapirus bairdii \\
\hline & \multirow{2}{*}{ Noctilionidae } & \multirow{2}{*}{$\begin{array}{l}\text { Noctilio albiventris } \\
\text { Noctilio leporinus }\end{array}$} & & & Tapirus pinchaque \\
\hline & & & \multirow{4}{*}{ Cetacea } & Balaenopteridae & Megaptera novaeangliae \\
\hline & Mormoopidae & Mormoops megalophylla & & Delphinidae & Sotalia fluviatilis \\
\hline & \multirow{11}{*}{ Phyllostomidae } & \multirow{11}{*}{$\begin{array}{l}\text { Anoura carishina } \\
\text { Anoura peruana } \\
\text { Carollia perspicillata } \\
\text { Enchisthenes hartii } \\
\text { Gardnerycteris crenulatum } \\
\text { Lonchorhina mankomara } \\
\text { Platyrrhinus chocoensis } \\
\text { Rhinophylla alethina } \\
\text { Sphaeronycteris } \\
\text { toxophyllum } \\
\text { Uroderma bakeri } \\
\text { Vampyrum spectrum } \\
\text { Vampyressa sinchi }\end{array}$} & & Iniidae & Inia geoffrensis \\
\hline & & & & Physeteridae & Physeter macrocephalus \\
\hline & & & \multirow{3}{*}{ Primates } & Aotidae & Aotus lemurinus \\
\hline & & & & Atelidae & Alouatta seniculus \\
\hline & & & & Cebidae & Cebus capucinus \\
\hline & & & \multirow{6}{*}{ Rodentia } & Sciuridae & $\begin{array}{l}\text { Microsciurus santanderensis } \\
\text { Sciurus igniventris }\end{array}$ \\
\hline & & & & Cricetidae & Nephelomys meridensis \\
\hline & & & & Caviidae & Hydrochoerus hydrochaeris \\
\hline & & & & Dasyproctidae & Dasyprocta punctata \\
\hline & & & & Cuniculidae & Cuniculus paca \\
\hline & & & & Erethizontidae & Coendou vestitus \\
\hline & Natalidae & Natalus tumidirostris & & & \\
\hline & Thyropteridae & Thyroptera tricolor & & & \\
\hline & Vespertilionidae & $\begin{array}{l}\text { Eptesicus andinus } \\
\text { Eptesicus miradorensis } \\
\text { Myotis keaysi }\end{array}$ & & & \\
\hline
\end{tabular}

Los registros de especímenes proveídos por las colecciones biológicas representaron información de 12 órdenes, 37 familias, 133 géneros y 246 especies. Al igual que en los registros de observaciones algunas entidades fueron identificados al nivel de género y se incluyeron especies invasoras. Más del $85 \%$ de los datos corresponden a individuos del orden Chiroptera, el segundo orden con más registros fue Rodentia y los demás órdenes tuvieron una representación inferior al 2\% (Tabla 5).

Geográficamente, los registros de colecciones incluyeron datos de 29 departamentos. Registros de los departamentos de Atlántico, Arauca, La Guajira, Guainía y Vaupés no fueron reportados por ninguna de las entidades vinculadas en el presente convenio (Figura 2a).

Si bien los compromisos del convenio correspondían a 3.500 registros, 50 fichas de especies y una lista de referencia de especies de mamíferos de Colombia, gracias al trabajo colaborativo de todos los actores involucrados obtuvimos 2.144 registros y 15 fichas de especies adicionales, así como una lista de especies de mamíferos actualizada con 528 reportes de mamíferos que ocurren en el país.

\footnotetext{
Mammalogy Notes | Notas Mastozoológicas

Sociedad Colombiana de Mastozoología

Vol. 4 Num. 2| 2018
} 


\section{Correspondencia | Correspondencia}

La red de trabajo construida es el producto más valioso obtenido. La ejecución del convenio fue exitosa debido al esfuerzo colaborativo, incluyente, dinámico y participativo de las universidades públicas y privadas, las colecciones mastozoológicas, las organizaciones no gubernamentales, la SCMas y sus respectivos socios. Todos unidos en pro del logro de cada uno de los objetivos encomendados por el IAvH.

Es necesario dar continuidad a los procesos de sistematización, estandarización, estructuración, georreferenciación, y curaduría taxonómica de los mamíferos de Colombia; aproximadamente 75.000 registros reposan en las colecciones biológicas del país, por lo que el $93 \%$ de esta cifra requiere de este mismo proceso.

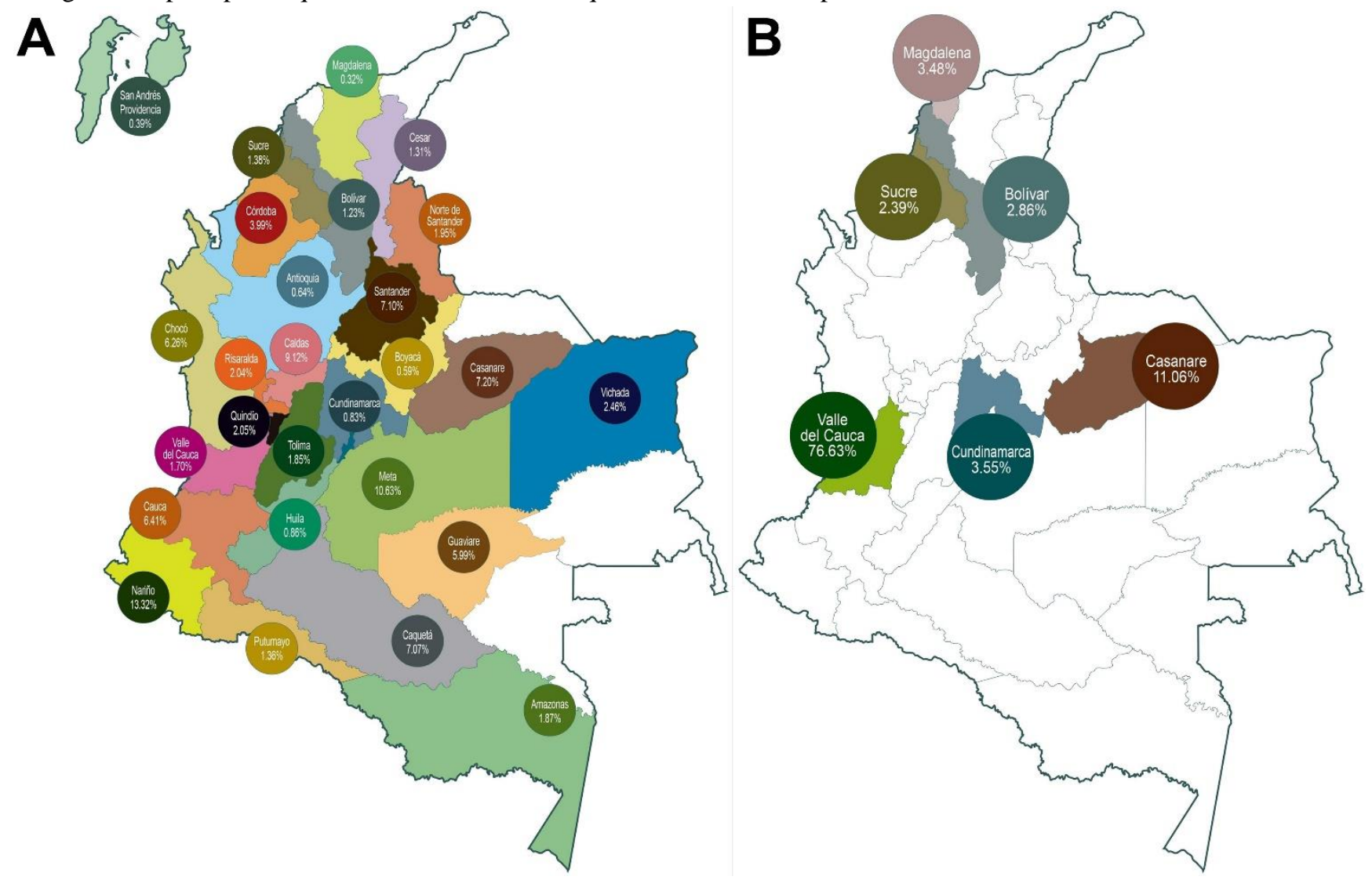

Figura 2. Porcentaje de representatividad geográfica de los registros de mamíferos de Colombia provenientes de: A) colecciones mastozoológicas, B) observaciones y fototrampeo.

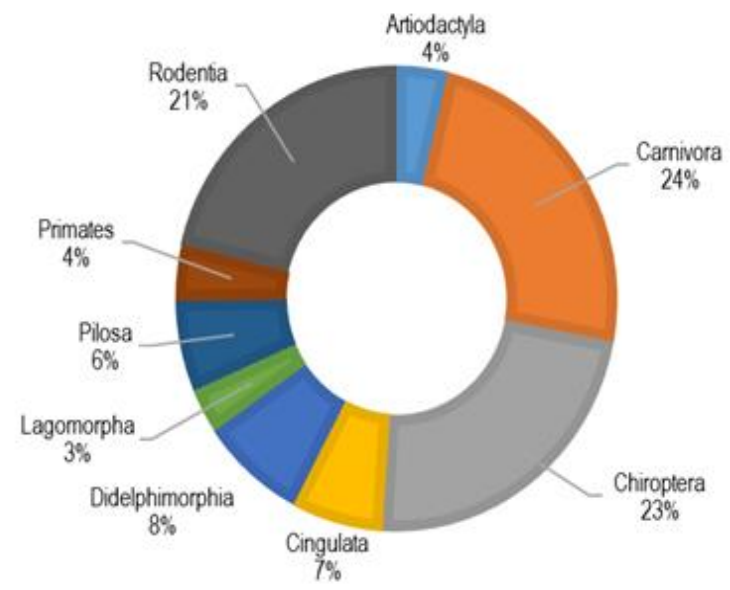

Figura 3. Porcentaje de registros observacionales en los órdenes de mamíferos para Colombia

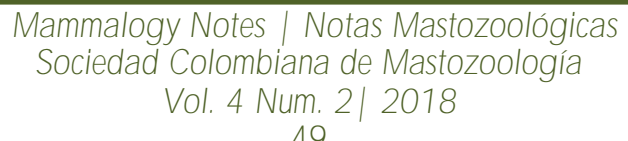


Tabla 4. Porcentaje de registros observacionales en los órdenes de mamíferos de Colombia

\begin{tabular}{|c|c|c|c|c|}
\hline Nombre de la Institución y colección & Sigla & $\begin{array}{l}\text { Tipo de } \\
\text { Registros }\end{array}$ & $\begin{array}{c}\text { Número de } \\
\text { Registros }\end{array}$ & $\begin{array}{l}\text { Porcentaje } \\
\text { de registros }\end{array}$ \\
\hline Universidad Nacional de Colombia, Colección de Mamíferos "Alberto Cadena García" & ICN & Colección & 252 & 4,46 \\
\hline Universidad del Valle, Colección de Mamíferos & UV & Colección & 262 & 4,64 \\
\hline Universidad de la Amazonía, Museo de Historia Natural, Colección de Mamíferos & UAM - M & Colección & 250 & 4,42 \\
\hline Universidad del Quindío, Colección de Mamíferos & CMUQ & Colección & 301 & 5,33 \\
\hline Universidad de La Salle, Museo de La Salle, Colección de Mamíferos & qui & Colección & 252 & 4,46 \\
\hline Universidad del Cauca, Museo de Historia Natural, Colección Mastozoológica & MHNUC- M & Colección & 251 & 4,44 \\
\hline Universidad Industrial de Santander, Museo de Historia Natural, Colección Mastozoológica & UIS-MHN & Colección & 287 & 5,08 \\
\hline Universidad Tecnológica del Chocó, Colección Teriológica del Chocó & $\mathrm{CMCH}$ & Colección & 250 & 4,42 \\
\hline Universidad de Antioquia, Colección Teriológica Universidad de Antioquia & CTUA & Colección & 272 & 4,81 \\
\hline Universidad de Nariño, Colección Zoológica & PSO-CZ & Colección & 551 & 9,76 \\
\hline Universidad de Caldas, Museo de Historia Natural, Colección de Mamíferos & MHN-UCa & Colección & 260 & 4,60 \\
\hline $\begin{array}{l}\text { Universidad Distrital Francisco José de Caldas, Museo de Historia Natural, Colección de } \\
\text { Mamíferos }\end{array}$ & MUD & Colección & 276 & 4,89 \\
\hline Universidad de los Llanos, Museo de Historia Natural, Colección de Mamíferos & MHNU-M & Colección & 251 & 4,44 \\
\hline $\begin{array}{l}\text { Universidad Pontificia Javeriana, Museo Javeriano de Historia Natural Lorenzo Uribe } \\
\text { Uribe, Colección de Mamíferos }\end{array}$ & MPUJ-Mamm & Colección & 252 & 4,46 \\
\hline Corporación para la Gestión Ambiental Biodiversa & Biodiversa & Observaciones & 1.122 & 19,87 \\
\hline Fundación Proyecto de Conservación de Aguas y Tierras - ProCAT Colombia & ProCAT & Observaciones & 253 & 4,48 \\
\hline Fundación Reserva Natural La Palmita & FRNLP & Colección & 213 & 3,77 \\
\hline Fundación Reserva Natural La Palmita & FRNLP & Observaciones & 89 & 1,57 \\
\hline TOTAL DE REGISTROS & & & 5.644 & 100 \\
\hline
\end{tabular}

Tabla 5. Número y porcentaje de registros de colecciones por órdenes de mamíferos para Colombia

\begin{tabular}{lcc}
\hline \hline \multicolumn{1}{c}{ Orden } & Número de registros & Porcentaje \\
\hline Chiroptera & 3.571 & 85,53 \\
\hline Rodentia & 371 & 8,88 \\
\hline Artiodactyla & 64 & 1,53 \\
\hline Didelphimorphia & 52 & 1,24 \\
\hline Primates & 34 & 0,81 \\
\hline Carnivora & 37 & 0,88 \\
\hline Pilosa & 15 & 0,35 \\
\hline Lagomorpha & 11 & 0,26 \\
\hline Cetacea & 6 & 0,14 \\
\hline Cingulata & 6 & 0,14 \\
\hline Paucituberculata & 6 & 0,14 \\
\hline Soricomorpha & 2 & 0,04 \\
\hline \hline
\end{tabular}

\section{Agradecimientos}

Este convenio fue producto del esfuerzo de organizaciones y personas que destinaron su tiempo, recursos y conocimiento, de manera generosa para mejorar y divulgar la información disponible de los mamíferos de Colombia. La Sociedad Colombiana de Mastozoología quiere resaltar la labor de:

\section{COLOMBIA BIO}

Felipe García Cardona | Gerente Colombia BIO

\section{INSTITUTO ALEXANDER von HUMBOLDT}

Brigitte Baptiste | Directora Instituto Humboldt

Hernando García | Subdirector Científico Instituto Humboldt

Dairo Escobar | Coordinador $\mathrm{SiB}$

Leonardo Buitrago | Líder - Administración de Contenidos SiB

Javier Gamboa | Líder - Productos y Servicios

Sandra Peña | Profesional Junior, Programa de Evaluación y Monitoreo

Jeimy Patricia Díaz Rodríguez | Auxiliar Administrativo, Programa Evaluación y Monitoreo del Estado de la Biodiversidad

\section{SOCIOS Y COORDINADORES DE COMITÉS DE TRABAJO DE LA SCMaS}

Andrés Felipe Suárez Castro | Coordinador Comité de Comunicaciones

Dora Catalina Concha | Coordinadora Comité de Educación y Desarrollo

Angélica Trujillo | Coordinadora Comité de Educación y Desarrollo

Elkin Noguera Urbano | Coordinador Comité de Investigaciones

Jairo Pérez Torres | Coordinador Comité Ética

Miguel Eduardo Rodríguez | Coordinador Comité de Ética

José F. González Maya | Coordinador Comité Editorial

Alexander Velásquez Valencia | Coordinador Comité de eventos

\section{Mammalogy Notes | Notas Mastozoológicas \\ Sociedad Colombiana de Mastozoología \\ Vol. 4 Num. 2| 2018}




\section{Correspondencia | Correspondencia}

AUXILIAR ADMINISTRATIVO

Carolina López Castañeda

JUNTA DIRECTIVA SOCIEDAD COLOMBIANA DE MASTOZOOLOGIA

Danny Zurc | Presidente (e)

Hugo Mantilla Meluk | Representante legal, Vicepresidente (e)

Mauricio Vela | Secretario (e)

Gabriel Pantoja | Tesorero (e)

Juan David Sánchez | Vocal representante de los académicos (e)

Darwin Morales | Vocal representante estudiantil (e)

Lorena A. Marín-Carvajal | Fiscal (e)

\section{CURADORES DE COLECCIONES BIOLÓGICAS}

\begin{tabular}{ll}
\hline \hline \multicolumn{1}{c}{ Curador } & \multicolumn{1}{c}{ Institución, Nombre de Colección } \\
\hline Abelardo Rodríguez-Bolaños & Universidad Distrital Francisco José de Caldas, Museo de Historia Natural, Colección de Mamíferos \\
Alexander Velásquez Valencia & Universidad de la Amazonía, Museo de Historia Natural, Colección de Mamíferos \\
Cristian Cruz-Rodríguez & Universidad de La Salle, Museo de La Salle, Colección de Mamíferos \\
Francisco Sánchez & Universidad de los Llanos, Museo de Historia Natural, Colección de Mamíferos \\
Héctor Ramírez Chaves & Universidad de Caldas \\
Hugo López-Arévalo & Universidad Nacional de Colombia, Colección de Mamíferos "Alberto Cadena García" \\
Hugo Mantilla-Meluk & Universidad del Quindío, Colección de Mamíferos \\
Jairo Pérez-Torres & Pontificia Universidad Javeriana, Museo Javeriano de Historia Natural Lorenzo Uribe Uribe, Colección de Mamíferos \\
Jhon Jairo Calderón Leyton & Universidad de Nariño, Colección Zoológica \\
María del Pilar Rivas Pava & Universidad del Cauca, Museo de Historia Natural, Colección Mastozoológica \\
Martha Patricia Ramírez & Universidad Industrial de Santander, Museo de Historia Natural, Colección Mastozoológica \\
Orfelina Rios Medina & Universidad Tecnológica del Chocó, Colección Teriológica del Chocó \\
Oscar Murillo & Universidad del Valle, Colección de Mamíferos \\
Sergio Solari & Universidad de Antioquia, Colección Teriológica U. de A. \\
\hline \hline
\end{tabular}

FACILITADORES DE Organizaciones No Gubernamentales

\begin{tabular}{ll}
\hline \hline \multicolumn{1}{c}{ Curador } & \multicolumn{1}{c}{ Institución } \\
\hline Andrea Cáceres & Corporación para la Gestión Ambiental Biodiversa \\
José F. González-Maya & Fundación Proyecto de Conservación de Aguas y Tierras - ProCAT Colombia \\
Miguel Rodríguez-Posada & Fundación Reserva Natural La Palmita \\
\hline \hline
\end{tabular}

\section{ESTUDIANTES-BECARIOS}

\begin{tabular}{ll}
\hline \hline \multicolumn{1}{c}{ Nombre } & \multicolumn{1}{c}{ Institución, Nombre de Colección } \\
\hline Alejandra Bonilla & Universidad de Antioquia, Colección Teriológica U. de A. \\
Alejandra Hurtado & Corporación para la Gestión Ambiental Biodiversa \\
Alfaro Asprilla & Universidad Tecnológica del Chocó, Colección Teriológica del Chocó \\
Angie Solorzano & Universidad Distrital Francisco José de Caldas, Museo de Historia Natural, Colección de Mamíferos \\
Camilo Casas & Proyecto de Conservación de Aguas y Tierras - ProCAT Colombia \\
Christian David Cabrera Ojeda & Universidad de Nariño, Colección Zoológica \\
Darly Gabriela Muñoz Lara & Universidad del Cauca, Museo de Historia Natural, Colección Mastozoológica \\
Diana Ramírez & Universidad de los Llanos, Museo de Historia Natural, Colección de Mamíferos \\
Diego Rolando Gutiérrez Sanabria & Fundación Reserva Natural La Palmita \\
Edwar Fabián Farfán Espinosa & Universidad de la Amazonía, Museo de Historia Natural, Colección de Mamíferos \\
Ingrith Y. Mejía Fontecha & Universidad de Caldas, Museo de Historia Natural, Colección de Mamíferos |Colegio Mayor del Cauca \\
Apoya Karime Alejandra Gómez B. & Universidad Industrial de Santander, Museo de Historia Natural, Colección Mastozoológica \\
Javier E. Colmenares & Universidad Nacional de Colombia, Colección de Mamíferos "Alberto Cadena García" \\
Jennifer Zilentsijgh & Universidad de La Salle, Museo de La Salle, Colección de Mamíferos \\
Juliet Cárdenas & Universidad del Quindío, Colección de Mamíferos \\
Leidy Johana Sánchez & Universidad del Valle, Colección de Mamíferos \\
Luisa Alejandra Ruano & Pontificia Universidad Javeriana, Museo Javeriano de Historia Natural Lorenzo Uribe Uribe, Colección de Mamíferos \\
Sandra Milena Chala Quintero &
\end{tabular}

\footnotetext{
Mammalogy Notes | Notas Mastozoológicas

Sociedad Colombiana de Mastozoología

Vol. 4 Num. 2| 2018
} 
Autores proveedores de contenido de fichas de especies.

\begin{tabular}{|c|c|c|c|}
\hline Nombre & Afiliación & $\begin{array}{l}\text { Rol en el } \\
\text { Convenio }\end{array}$ & Aportes \\
\hline A. L. Pulido González & Universidad del Quindío & I & Cebus capucinus \\
\hline Alicia Alexandra Pineda Guerrero & University Wisconsin Madison & I & Puma yagouaroundi, Leopardus tigrinus \\
\hline Andrés Arias - Alzate & Grupo de Mastozoología Universidad de Antioquia & I & Tapirus pinchaque, Lotra longicaudis, Panthera onca, Puma concolor, Eira barbara, Cerdocyon thous \\
\hline Andrés Vargas Arboleda & Universidad del Quindío & I & Myotis keaysi, Anoura peruana, Anoura carishina \\
\hline Angela María Villamizar Ramírez & Universidad Industrial de Santander & I & Nephelomys meridensis \\
\hline Angélica Trujillo Acosta & Independiente & I & Choloepus hoffmanni, Cuniculus paca \\
\hline Angie N. Tinoco Sotomayor & Universidad de Cartagena & I & Dasyprocta punctata, Cuniculus paca, Choloepus hoffmanni, Bradypus variegatus \\
\hline C. Peña Vergara & Universidad del Quindío & I & Cebus capucinus \\
\hline Camilo Ernesto Angarita Yanes & Universidad de Pamplona & I & Nephelomys meridensis \\
\hline Camilo Paredes & ProCAT Colombia & I & Galictis vittata \\
\hline Carlos A. Delgado V & Universidad CES & I & Cerdocyon thous \\
\hline Carlos Andrés Aya Cuero & $\begin{array}{l}\text { Museo de La Salle } \\
\text { Universidad Distrital Francisco José de Caldas }\end{array}$ & I & $\begin{array}{c}\text { Vampyrum spectrum, Priodontes maximus, Leopardus pardalis, Dasypus sabanicola, Dasypus novemcinctus, } \\
\text { Dasypus kappleri, Cabassous unicinctus, Cabassous centralis }\end{array}$ \\
\hline Carlos H Cáceres Martínez & Universidad Nacional, Medellín & I & Hydrochoerus hydrochaeris, Tamandua mexicana \\
\hline Cesar Rojano Bolaño & Cunaguaro & I & Tamandua mexicana, Myrmecophaga tridactyla \\
\hline Cristian Cruz-Rodríguez & Museo La Salle & $\mathrm{F}$ & $\begin{array}{c}\text { Priodontes maximus, Leopardus wiedii, Natalus tumidirostris, Mormoops megalophylla, Leopardus pardalis, Carolia } \\
\text { perspicillata }\end{array}$ \\
\hline D. Caicedo Herrera & Omacha & I & Trichechus inunguis \\
\hline Dennis Castillo Figueroa & Pontificia Universidad Javeriana & I & Rhynchonycteris naso \\
\hline Diana Katherine Camelo Pinzón & Universidad Distrital Francisco José de Caldas & I & Vampyrum spectrum \\
\hline Diana Marcela Ramirez Castellanos & Universidad de los Llanos & B & Gardnerycteris crenulatum \\
\hline Diego Alejandro Esquivel Melo & Universidad Distrital Francisco José de Caldas & I & Vampyrum spectrum \\
\hline Diego J. Lizcano & TNC & I & Tapirus pinchaque \\
\hline Diego Rolando Gutiérrez-Sanabria & Fundación Reserva Natural La Palmita & B & Sphaeronycteris toxophyllum \\
\hline Edward Fabián Farfán Espinosa & Universidad de la Amazonía & B & Urosciurus igniventris \\
\hline Elkin A. Noguera Urbano & Universidad Nacional Autónoma de México & I & Epstesicus miradorensis \\
\hline Federico Mosquera Guerra & Fundación Omacha & I & Sotalia fluviatilis, Pteronura brasiliensis, Inia geoffrensis, Trichechus inunguis \\
\hline Fernando Trujillo & Fundación Omacha & I & Sotalia fluviatilis, Inia geoffrensis, Trichechus inunguis \\
\hline Gabriel Ernesto Pantoja Peña & Pontificia Universidad Javeriana & I & Natalus tumidirostris, Mormoops megalophylla, Carolia perspicillata \\
\hline Gina Olarte & Corporación Universitaria de Santa Rosa de Cabal & I & Speothos venaticus \\
\hline $\begin{array}{l}\text { Grupo de Inv. Manejo y Gestión de } \\
\text { la Vida Silvestre del Chocó }\end{array}$ & Colección Ter. Universidad Tecnológica del Chocó & $\mathrm{F}$ & Rhinophylla alethina, Platyrrihus chocoensis \\
\hline Héctor Andrés Hernández Pinson & & I & Thyroptera tricolor, Noctilio albiventris \\
\hline Héctor E. Ramírez Chaves & Universidad del Cauca & $\mathrm{F}$ & Vampyressa sinchi, Epstesicus miradorensis, Coendou vestitus \\
\hline Hugo A Vides - Avilez & Universidad de Cartagena & I & Dasyprocta punctata, Cuniculus paca \\
\hline Hugo Fernando López-Arévalo & Colección de Mamíferos Alberto Cadena García & $\mathrm{F}$ & Procyon lotor \\
\hline Hugo Mantilla-Meluk & Universidad del Quindío & $\mathrm{F}$ & Anoura peruana, Anoura carishina, Uroderma bakeri, Lonchorhina mankomara \\
\hline Isabel Cristina Avila & Universidad de Friburgo & I & Physeter macrocephalus, Megaptera novaeangliae \\
\hline J. Sebastián Jiménez Alvarado & Procat & I & Galictis vittata \\
\hline Jairo Pérez-Torres & Pontificia Universidad Javeriana & $\mathrm{F}$ & Natalus tumidirostris, Mormoops megalophylla, Carolia perspicillata \\
\hline Javier Enrique Colmenares Pinzón & Universidad Industrial de Santander & B & Microsciurus santanderensis \\
\hline José F. González-Maya & ProCAT Colombia & $\mathrm{F}$ & Tamandua mexicana, Panthera onca, Puma concolor, Leopardus pardalis, Galictis vittata, Eira barbara \\
\hline José Fernando Navarro Peláez & Universidad de Antioquia & I & Puma concolor \\
\hline Juan Camilo Cepeda Duque & Corporación Universitaria de Santa Rosa de Cabal & I & Speothos venaticus \\
\hline Juan David Sánchez-Londoño & Universidad CES & I & Bassaricyon neblina \\
\hline Juan Felipe Albarracín Caro & Pontificia Universidad Javeriana & I & Rhynchonycteris naso \\
\hline Julio Chacón Pacheco & Universidad de Córdoba & I & $\begin{array}{l}\text { Priodontes maximus, Cyclopes didactylus, Tamandua mexicana, Myrmecophaga tridactyla, Dasypus sabanicola, } \\
\text { Dasypus novemcinctus, Dasypus kappleri, Cabassous unicinctus, Choloepus hoffmanni, Cabassous centralis }\end{array}$ \\
\hline Karime A- Gómez-B & Institución Universitaria Colegio Mayor del Cauca & B & Vampyressa sinchi \\
\hline Keiner Meza - Tilvez & Universidad de Cartagena & I & Choloepus hoffmanni, Bradypus variegatus \\
\hline Kevin González Gutiérrez & Universidad del Tolima & I & Noctilio leporinus \\
\hline Luisa Alejandra Ruano Meneses & Universidad del Valle & B & Enchisthenes hartii \\
\hline Luisa Fernanda Arcila Pérez & Universidad del Quindío & I & Aotus lemurinus \\
\hline M. A. Bedoya Cañón & Universidad del Quindío & I & Cebus capucinus \\
\hline Manuela Montoya Marín & Corporación Universitaria de Santa Rosa de Cabal & I & Tapirus pinchaque \\
\hline María Alejandra Hurtado Materón & Universidad del Valle- Fund. Biodiversa & B & Caenolestes fuliginosus \\
\hline María Camila Dueñas Cuellar & Institución Universitaria Colegio Mayor del Cauca & I & Vampyressa sinchi \\
\hline Maria Camila Latorre Cárdenas & Instituto de Biología UNAM & I & Lontra longicaudis \\
\hline María M. Torres-Martínez & Unversidade Federal do Paraná & I & Epstesicus miradorensis, Coendou vestitus \\
\hline Mariana Vélez Orozco & Corporación Universitaria de Santa Rosa de Cabal & I & Eptesicus andinus \\
\hline Nerieth Goretti Leuro & Museo de la Salle & I & Leopardus wiedii \\
\hline Paula Catalina Pinilla Cortes & Universidad Distrital Francisco José de Caldas & I & Vampyrum spectrum \\
\hline Sandra Milena Chala Quintero & Pontificia Universidad Javeriana & B & Natalus tumidirostris, Mormoops megalophylla, Carolia perspicillata \\
\hline Sebastian Botero & Grupo Mastozoología Universidad de Antioquia & I & Puma yagouaroundi, Leopardus tigrinus \\
\hline Sebastián Cuadrado Rios & Universidad del Quindío, Universidad Nacional & I & Anoura peruana \\
\hline Sebastián Mejía Correa & Independiente & I & Tapirus bairdii \\
\hline Sebastián O. Montilla & Universidad del Quindío & I & Aotus lemurinus \\
\hline Sergio Solari & Universidad de Antioquia & $\mathrm{F}$ & Marmosa isthmica \\
\hline
\end{tabular}

Rol en el convenio: I: Investigador, B: Becario convenio, F: Facilitador

Y a todos los que hicieron parte de este proceso. GRACIAS. 


\section{Correspondencia | Correspondencia}

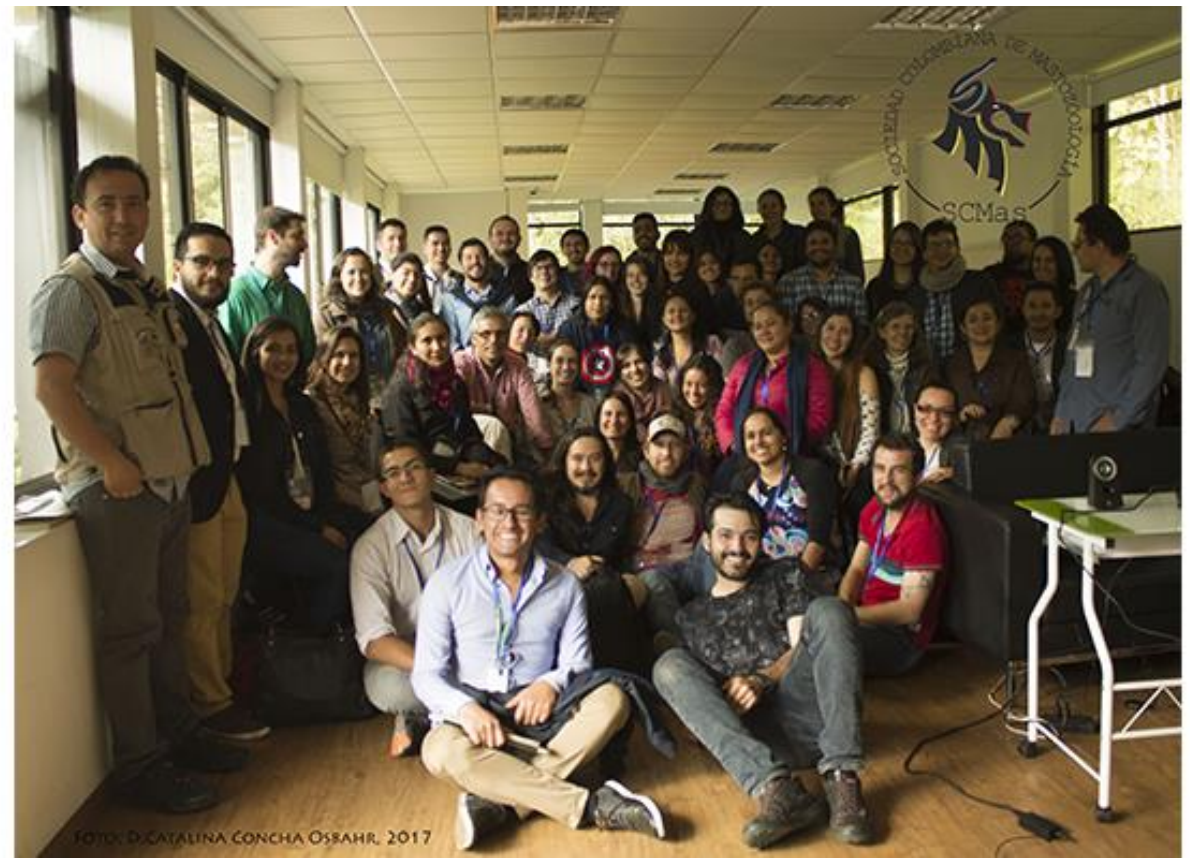

Foro Mamíferos de Colombia

(Fotografía por Dora Catalina Concha Osbahr)

Referencias

SUÁREZ-CASTRO, A. F. \& H. E. RAMÍREZ-CHAVES. 2014. Adiciones y cambios a la lista de mamíferos de Colombia: 500 especies registradas para el territorio nacional. Mammalogy Notes (1-2):223-235.

RAMÍREZ-CHAVES H., F. SUÁREZ-CASTRO \& J.F. GONZÁLEZ-MAYA. 2016. Cambios recientes a la lista de mamíferos de Colombia. Mammalogy Notes 3(1):1-20.

SOLARI S., Y. MUÑOZ-SABA, J. V. RODRÍGUEZ-MAHECHA, T. R. DEFLER, H. E. RAMÍREZ-CHAVES \& F. TRUJILLO. 2013. Riqueza, endemismo y conservación de los mamíferos de Colombia. Mastozoología Neotropical 20(2):301 - 365. 